\title{
Comparación entre el Método Directo y la Fórmula de Friedewald para Determinar los Niveles de Colesterol LDL en Caninos
}

\author{
Comparison between the Direct Method and the Friedewald Formula to Measure \\ LeVels of LDL ChOlesterol in Dogs
}

\author{
José Henry Osorio ${ }^{1,2}$, Yirly Johanna Suárez ${ }^{1}$, Jazmín Vinasco ${ }^{1}$
}

\section{Resumen}

\begin{abstract}
El objetivo del estudio fue comparar el método Directo con la fórmula de Friedewald para la determinación de colesterol LDL en caninos. Se tomaron 185 muestras de sangre de caninos adultos en ayuno y sin restricción de sexo. Se extrajo el suero y se determinaron los niveles de colesterol LDL con los dos métodos. El promedio, desviación estándar y valores mínimos y máximos por el método Directo fueron de 49.2 $\pm 16.7,14.6$ y $96.0 \mathrm{mg}$ / dl, respectivamente, y por la fórmula de Friedewald fueron de $65.2 \pm 31.5,4.6$ y $184.2 \mathrm{mg} /$ $\mathrm{dl}$, respectivamente, habiendo diferente estadística $(\mathrm{p}<0.05)$ entre métodos. Se recomienda el uso del método directo para la determinación de los niveles de colesterol LDL en caninos.
\end{abstract}

Palabras clave: colesterol LDL, perro, método directo, fórmula de Friedewald

\section{Abstract}

The aim of this study was to compare the Direct method and the Friedewald formula method for the determination of LDL cholesterol in dogs. Blood samples were collected in 185 fasted dogs without sex restriction. Serum was extracted and levels of LDL cholesterol were determined by the two methods. The mean, standard deviation, and minimum and maximum values by the Direct method were $49.2 \pm 16.7,14.6$ and $96.0 \mathrm{mg} / \mathrm{dl}$, respectively, and by the Friedewald method were $65.2 \pm 31.5,4.6$ and $184.2 \mathrm{mg} / \mathrm{dl}$, respectively, with significant difference between the methods $(\mathrm{p}<0.05)$. The Direct method is recommended for determining LDL cholesterol levels in dogs.

Key words: LDL cholesterol, canine, direct method, friedewald method

\footnotetext{
${ }^{1}$ Laboratorio de Bioquímica Clínica y Patología Molecular, Departamento de Ciencias Básicas de la Salud, Universidad de Caldas, Manizales, Colombia

${ }^{2}$ E-mail: jose.osorio_o@ucaldas.edu.co
} 


\section{INTRODUCCIÓN}

Las lipoproteínas (LP) son macromoléculas esféricas que presentan una base o núcleo hidrofóbico o no polar dirigido hacia el interior, y contienen triglicéridos y ésteres de colesterol responsables de exponer la molécula hacia donde ocurre el metabolismo, siendo el transporte de lípidos una de sus funciones principales (Osorio, 2006; Puppione et al., 2008; Osorio et al., 2010; Xenouli y Steiner, 2010). Se clasifican, según su densidad, en quilomicrones $(\mathrm{Q})$, lipoproteínas de muy baja densidad (VLDL), lipoproteínas de baja densidad (LDL) y lipoproteínas de alta densidad (HDL) (Osorio y Giraldo, 1999; Pasquini et al., 2008; Mori et al., 2010).

El metabolismo de las lipoproteínas difiere según la especie. Así, en caninos, felinos, equinos, bovinos y ratones predominan las HDL, dándoles el nombre de mamíferos HDL, en contraste con el humano, cerdos, monos, conejos, cobayos, hámster y otros de vida silvestre donde predomina el colesterol LDL (Maldonado et al., 2001; Coppo et al., 2003). En el humano, el consumo de alimentos ricos en colesterol genera un aumento en los niveles de colesterol LDL, conllevando a un mayor riesgo de enfermedades cardiovasculares y ateroesclerosis (Colpo, 2005; Laris et al., 2005). No obstante, en el canino, las HDL ayudan a eliminar el exceso de apoproteínas y trasladan el colesterol de los tejidos extrahepáticos al hígado, mediante el proceso de transporte inverso del colesterol, el cual se considera esencial para el mantenimiento del equilibrio de los esteroles, reduciendo el riesgo aterogénico en esta especie (Osorio et al., 2010).

Son pocos los estudios en caninos que permiten estandarizar un método que determine el colesterol LDL, haciendo difícil la interpretación de los resultados al momento de realizar diagnósticos de enfermedades metabólicas. Es por ello que el objetivo del presente estudio fue comparar el método directo con la fórmula de Friedewald en la de- terminación de los niveles de colesterol LDL en caninos.

\section{Materiales y Métodos}

Se trabajó con 185 caninos adultos, sin restricción de sexo. Los canes se encontraban en ayunas, con un plan sanitario adecuado (desparasitados, vacunados) y clínicamente sanos.

Las muestras se obtuvieron mediante punción de la vena yugular en tubos Vacutainer ${ }^{\circledR} \sin$ anticoagulante. Se llevaron al laboratorio en condiciones de refrigeración y fueron centrifugadas a $2300 \mathrm{~g}$ por $15 \mathrm{~min}$. Con el suero resultante se determinaron los niveles de colesterol LDL mediante el método directo (Tietz, 1991), utilizando los reactivos comerciales BioSystems ${ }^{\circledR}$, EEUU. Aquí, el colesterol HDL es precipitado en presencia de ácido fosfotúngstico y determinado mediante el método enzimático-colorimétrico aplicado para la determinación del colesterol total de la muestra. Adicionalmente, se determinaron los niveles de triglicéridos mediante un método enzimático-colorimétrico (BioSystems ${ }^{\circledR}$, EEUU). En la lectura de los resultados se utilizó un analizador semiautomático de química serial marca Rayco (IHR Diagnóstica, Colombia).

Los valores de los triglicéridos fueron divididos entre cinco, donde el valor resultante corresponde a los niveles de colesterol VLDL. Los valores de colesterol LDL fueron calculados restando el colesterol HDL y el colesterol VLDL del colesterol total (Friedewald et al., 1972).

Se determinó el promedio con su desviación estándar y los valores mínimos y máximos para la cuantificación del colesterol LDL, tanto directo como para la fórmula de Friedewald. Se evaluaron las diferencias entre los dos métodos por medio de un análisis de varianza simple, utilizando el programa Statgraphics Plus 5.1. 
Cuadro 1. Niveles de colesterol LDL en caninos, determinados por el método directo y por la fórmula de Friedewald

\begin{tabular}{lcccc}
\hline Método & Promedio & $\begin{array}{c}\text { Desviación } \\
\text { estándar }\end{array}$ & Mínimo & Máximo \\
\hline Directo & $49.2^{\mathrm{a}}$ & 16.7 & 14.6 & 96.0 \\
Fórmula de Friedewald & $65.2^{\mathrm{b}}$ & 31.5 & 4.6 & 184.2 \\
\hline
\end{tabular}

${ }^{a, b}$ Superíndices diferentes indican diferencia estadística $(p<0.05)$

\section{Resultados y Discusión}

Los niveles de colesterol LDL determinados por los dos métodos se muestran en el Cuadro 1. Los resultados contradicen varios estudios realizados en otras especies animales con el mismo patrón HDL como el equino (Osorio y Uribe-Velázquez, 2011) y el bovino (Osorio et al., 2012b), donde no se encontraron diferencias al comparar ambos métodos, validando la fórmula de Friedewald. En forma similar, Osorio et al. (2012a), en un estudio en pollos de engorde recomendaron el método directo para la medición de colesterol LDL, ya que el método precipitado y la fórmula de Friedewald pueden tener imprecisiones o errores al momento de realizar dicha medición.

Se han realizado investigaciones en humanos donde se ha comparado el método directo con la fórmula de Friedewald, obteniéndose valores más bajos con el método directo (Mendes de Cordova et al., 2004), lo cual coincide con los resultados del presente estudio. Además, indican que el método directo es el más apropiado para el laboratorio de rutina, ya que no tiene las interferencias que presenta el cálculo de la fórmula de Friedewald. En contraste, Querales et al. (2012) compara las tres técnicas utilizadas en el laboratorio clínico (determinación homogénea directa, fórmula de Friedewald y técnica de precipitación), infiriendo que hay mayor similitud entre los va- lores de colesterol LDL obtenidos por determinación directa y la fórmula de Friedewald, en comparación con aquellos entre la fórmula de Friedewald y el método de precipitación. Estos resultados coinciden con otros reportes en humanos (Miller et al., 2002; Sahu et al., 2005).

Los resultados permiten recomendar el uso del método directo para determinar los niveles de colesterol LDL en caninos.

\section{Agradecimiento}

Los autores agradecen a Jorge Enrique Pérez Cárdenas, del Departamento de Ciencias Básicas de la Salud de la Universidad de Caldas, por su tiempo y colaboración en la determinación de los perfiles lipídicos.

\section{Literatura Citada}

1. Colpo A. 2005. Colesterol LDL: ¿Colesterol «malo» o mala ciencia? J Am Phys S 10(3): 83-89.

2. Coppo NB, Coppo JA, Lazarte MA. 2003. Intervalos de confianza para colesterol ligado a lipoproteínas de alta y baja densidad en suero de bovinos, equinos, porcinos y caninos. Rev Vet 14(1): 1-8.

3. Friedewald WT, Levy RI, Fredrickson DS. 1972. Estimation of the concentration of low-density lipoprotein 
cholesterol in plasma, without use of the preparative ultracentrifuge. Clin Chem 18:499-502.

4. Laris MR, Arteaga A, Cuevas A, Rigotti A. 2005. El colesterol HDL: ¿un nuevo objetivo terapéutico en el manejo de las dislipidemias y la ateroesclerosis? Rev Med Chile 133: 823-832. doi: 10.4067/S0034-98872005000700011

5. Maldonado EN, Romero JR, Ochoa B, Aveldaño MI. 2001. Lipid and fatty acid composition of canine lipoproteins. Comp Biochem Physiol B Biochem Mol Biol 128: 719-729.

6. Mendes de Cordova C, Scheider CR, Juttel ID. 2004. Medición directa de LDL colesterol vs. F7. fórmula de Friedewald. Arq Bras Cardiol 83(6): 1-3.

7. Miller WG, Waymack PP, Anderson FP, Ethridge SF, Jayne EC. 2002. Performance of four homogeneous direct methods for LDL-cholesterol. Clin Chem 48(3): 489-98.

8. Mori N, Lee P, Muranaka S, Sagara $F$, Takemitsu H, Nishiyama Y, Yamamoto I, et al. 2010. Predisposition for primary hyperlipidemia in Miniature Schnauzers and Shetland sheepdogs as compared to other canine breeds. Res Vet Sci 88: 394-399. doi: 10.1016/ j.rvsc.2009.12.003

9. Osorio JH. 2006. Total cholesterol and HDL - cholesterol in aging dogs. Biosalud 5: 19-24.

10. Osorio JH, Giraldo CE. 1999. Perfil lipídico en caninos adultos obesos vs caninos adultos normales. Vet Zootec 11(1): 7-10.

11. Osorio J, Uribe-Velázquez L. 2011. Comparación de los métodos Directo y de Friedewald para la determinación de los niveles de colesterol LDL en el equino. Rev MVZ Córdoba 16: 2549-2553.

12. Osorio JH, Suárez YJ, UribeVelásquez LF. 2010. Metabolismo de los lípidos en caninos en el contexto de salud-enfermedad. Vet Zootec 4(1): 83-97.
13. Osorio JH, Flórez JD, Pérez JE. 2012a. Evaluación de los métodos directo, precipitado y Friedewald para la cuantificación de colesterol LDL y HDL en pollos de engorde. Rev Med Vet 24: 85-90.

14. Osorio JH, Suárez YJ, Pérez JE. $2012 b$. Validación de la fórmula de Friedewald para la determinación del perfil lipídico en bovinos. Biosalud 11(2): 70-76.

15. Pasquini A, Luchetti E, Cardini G. 2008. Plasma lipoprotein concentration in the dog: the effects of gender, age, breed and diet. J Anim Physiol Anim Nutr 92: 718-722. doi: 10.1111/j.14390396.2007.00771.x

16. Puppione DL, Bassilian S, Souda P, MacDonald MH, Hagland $F$, Whitelegge JP. 2008. Mass spectral analysis of the apolipoproteins on dog (Canis lupus familiaris) high density lipoproteins. Detection of apolipoprotein A-II. Comp Biochem Physiol Part D Genomics Proteomics 3: 290-296. doi: 10.1016/j.cbd.2008.08.002

17. Querales M, Cruces ME, Sánchez C, Querales M, Rojas S, Sánchez L. 2012. Medida del colesterol de lipoproteínas de baja densidad utilizando tres metodologías. Acta Bioquím Clín Latinoam 46(1): 31-38.

18. Sahu S, Chawla R, Uppal B. 2005. Comparison of two methods of estimation of low density lipoprotein cholesterol, the direct versus Friedewald estimation. Indian J Clin Biochem 20: 54-61. doi: 10.1007/BF02867401

19. Tietz NW. 1991. Clinical guide to laboratory tests. $2^{\text {nd }}$ ed. New York: Saunders. $1798 \mathrm{p}$.

20. Xenouli PG, Steiner JM. 2010. Lipid metabolism and hyperlipidemia in dogs. Vet J 183: 12-21. doi: 10.1016/ j.tvj1.2008.10.011 